\title{
ON THE MODULI SPACE OF POLYGONS IN THE EUCLIDEAN PLANE
}

\author{
MICHAEL KAPOVICH \& JOHN MILLSON
}

\begin{abstract}
We study the topology of moduli spaces of polygons with fixed side lengths in the Euclidean plane. We establish a duality between the spaces of marked Euclidean polygons with fixed side lengths and marked convex Euclidean polygons with prescribed angles.
\end{abstract}

1. We consider the space $\mathcal{P}_{n}$ of all polygons with $n$ distinguished vertices in the Euclidean plane $\mathbb{E}^{2}$ whose sides have nonnegative length allowing all possible degenerations of the polygons except the degeneration of the polygon to a point. Two polygons are identified if there exists an orientation preserving similarity of the complex plane $\mathbb{C}=\mathbb{E}^{2}$ which sends vertices of one polygon to vertices of another one. We shall denote the edges of the $n$-gon $P$ by: $e_{1}, \ldots, e_{n}$ and vertices by $v_{1}, \ldots, v_{n}$ so that $\vec{e}_{j}=v_{j+1}-v_{j}$. The space $\mathcal{P}_{n}$ is canonically isomorphic to the complex projective space $P(H)$ where $H \subset \mathbb{C}^{n}$ is the hyperplane given by

$$
H=\left\{\left(e_{1}, \ldots, e_{n}\right) \in \mathbb{C}^{n}: e_{1}+\ldots .+e_{n}=0\right\} .
$$

Therefore, the space $\mathcal{P}_{n}$ can be identified with $\mathbb{C} P^{n-2}$. The length of the edge $e_{j}$ will be denoted by $r_{j}$. We shall assume that all polygons are normalized so that the perimeter is equal to 1 .

Originally published in Volume 42, Number 1 without the figures. For the convenience of the reader, it is reprinted here with the figures. 
Let $\sigma$ be the conjugation $z \mapsto \bar{z}, z \in \mathbb{C}$. This transformation induces the involution $\sigma_{*}: \mathcal{P}_{n} \rightarrow \mathcal{P}_{n}$. The set of fixed points of $\sigma_{*}$ consists of all "degenerate polygons" $P$ which are contained in a straight line.

The map Area $: \mathcal{P}_{n} \rightarrow \mathbb{R}$ assigns to every normalized polygon its signed area.

Define the projection $\pi: \mathcal{P}_{n} \rightarrow \mathbb{R}^{n}$ which assigns to each normalized polygon $P \in \mathcal{P}_{n}$ the $n$-tuple of its side lengths :

$$
\pi(P)=r=\left(r_{1}, \ldots, r_{n}\right) .
$$

Our ultimate goal is to understand the topology of the moduli space of polygons with prescribed sides $M_{r}=\pi^{-1}(r), r \in \mathbb{R}^{n}$. We shall find necessary and sufficient conditions for $M_{r}$ to be connected, and describe the topology of the moduli spaces $M_{r}$ for $n=4,5,6$.

Theorem 1. The space $M_{r}$ is not connected if and only if there are 3 different sides $e_{i}, e_{j}, e_{k}$ in the normalized polygon so that

$$
r_{i}+r_{j}>1 / 2, \quad r_{i}+r_{k}>1 / 2, \quad r_{k}+r_{j}>1 / 2 .
$$

If $e_{i}, e_{j}, e_{k}$ exist as stated then $M_{r}$ is diffeomorphic to the disjoint union of two $(n-3)$-dimensional tori.

Remark 1. Roughly speaking Theorem 1 states that the moduli space of a polygon is disconnected if and only if it has "three long sides". To see examples with disconnected moduli spaces (and to motivate our result) start with a triangle- so that the moduli space consists of two points. Then cut off a small neighborhood of one of the vertices to create a quadrilateral which is (Hausdorff) close to the triangle. It is clear that the deformation space of such a quadrilateral has two components (for example use the function Area). Continue the process. Thus we obtain an $n$-gon with three long sides and disconnected moduli space. The fact that the moduli space consists of two $(n-3)$-dimensional tori is clear from Corollary 15: degenerate a short side and use induction.

Theorem 2. If the moduli space of pentagons $M_{r}$ is nonsingular, then $M_{r}$ is a compact oriented surface of the Euler characteristic $2(l(r)-3)$. Here $l(r)$ is the "level of the chamber" which contains the point $r \in \mathbb{R}^{5}$ (see Section 4, definition 2).

For example, the deformation space of the regular pentagon is a compact orientable surface of genus 4 .

Theorem 3. If the moduli space of hexagons $M_{r}$ is nonsingular and connected, then it is either diffeomorphic to a connected sum of $k$ 
copies of $\mathbb{S}^{2} \times \mathbb{S}^{1}$ and of the product $\Sigma_{g} \times \mathbb{S}^{1}$, or it is diffeomorphic to a connected sum of $T^{3} \# T^{3}$ and of $t$ copies of $\mathbb{S}^{2} \times \mathbb{S}^{1}$. Here $k \leq 4$, the genus $g$ of the surface $\Sigma_{g}$ is not greater than 4 and $t \leq 2$. If $M_{r}$ is nonsingular but is not connected, then it is diffeomorphic to the disjoint union of two copies of $T^{3}=\mathbb{S}^{1} \times \mathbb{S}^{1} \times \mathbb{S}^{1}$.

We will also show that for $n \geq 5$ the function $\operatorname{sign}\left(\right.$ Area) on $M_{r}$ fails to distinguish different connected components of $M_{r}$ (see Section 10).

In Sections 16- 19 we establish a duality $\tau$ between the spaces of Euclidean polygons with prescribed side lengths and marked convex polygons with prescribed angles. Unlike the classical duality in the spherical geometry, the correspondence $\tau$ is transcendental- it is given by hypergeometric integrals. Another difference is that the duality of polygons in $\mathbb{S}^{2}$ is local: it is enough to know two adjacent vertices of $P$ to find the corresponding vertex of the dual polygon $P^{*}$. It is not so in the case of Euclidean polygons: one has to know the whole polygon $P \subset \mathbb{C}$ to calculate a single vertex of the dual polygon $\tau(P)$. As a consequence of this duality we construct a piecewise-geodesic embedding $\mathcal{D} M$ of the moduli space $\bar{M}_{r}=M_{r} / \sigma$ into the complex-hyperbolic space $\mathbb{H}_{\mathbb{C}}^{n-3}$ for generic values of $r$. Points of $M_{r} / \sigma$ where the image of $\mathcal{D} M$ fails to be totally-geodesic correspond to polygons in $M_{r}$ which have parallel edges.

In our forthcoming work we shall discuss the relation of the moduli spaces of polygons with toric varieties, Hodge theory for representations of reflection groups and bending deformations of representations of polygons of groups. Results of Sections 2- 15 of our paper admit straightforward generalization to the case of polygons on the hyperbolic plane and unit sphere. In the last case it would be necessary to restrict consideration to polygons with perimeter not greater than $2 \pi$.

\section{Previous results}

After we had finished our paper we learned that many of our results were already known. Theorem 1 is proved in [12] and [18], is implicit in [16] and was known to Connelly. Theorem 2 is implicit in [12] and [16]. The result that the deformation space of a regular pentagon is a genus 4 surface was obtained independently in [11], [12], [13] and [16]. Our results concerning the Morse theory of lengths of diagonals (see Lemma 
11) are to be found in [10] and [12]. In [16], Walker gives an intrinsic description of the homology classes added after "crossing a wall" in terms of moduli spaces of the original polygon. He also makes an interesting conjecture: suppose that $r$ and $r^{\prime}$ lie in chambers which are not related by a permutation. Then $M_{r}$ and $M_{r^{\prime}}$ have different homology rings. In [1], Banchoff treates the Morse theory of the algebraic area function (the case of regular pentagons was considered in [11]). Some other aspects of moduli spaces of Euclidean and spherical polygons are discussed in [2], [7], [8].

2. First of all we have to understand the image of the projection $\pi: \mathcal{P}_{n} \rightarrow \mathbb{R}^{n}$. The image of this map is contained in the standard simplex $S$ in $\mathbb{R}^{n}$, which is equal to the intersection of the hyperplane $\Pi=\left\{r_{1}+\ldots+r_{n}=1\right\}$ with the first octant:

$$
r_{1} \geq 0, \ldots, r_{n} \geq 0 .
$$

Any point in the image of the map $\pi$ must satisfy the "triangle inequalities" :

$$
r_{j} \leq 1 / 2, \quad j=1, \ldots, n .
$$

Lemma 1. The inequalities (1) and (2) completely describe the domain $D_{n}=\pi\left(\mathcal{P}_{n}\right)$ in $\Pi$.

Proof. Let us prove this lemma by induction. For $n=3$ the assertion of Lemma is obviously correct. Now, assume that the assertion is correct for $n-1$. Suppose that a vector $r=\left(r_{1}, \ldots, r_{n}\right)$ satisfies the inequalities (1), (2). If the sum of lengths of each pair of adjacent edges $r_{i}+r_{i+1}$ is greater than $1 / 2$, then $r_{1}+\ldots+r_{n}>1$ which contradicts our assumptions. Hence, up to renumeration of vertices we have: $r_{n-1}+r_{n} \leq 1 / 2$. Therefore, according to the induction hypothesis, there exists an $n-1$-gon $P$ (with the perimeter 1 ) which has sides $\left(r_{1}, r_{2}, \ldots, r_{n-1}+r_{n}\right)$. Now, if we consider $P$ as an element of $\mathcal{P}_{n}$ with one extra vertex on $e_{n-1}$, then $\pi(P)=r$.

We can think about truncation of $S$ by the inequalities (2) as follows. For each vertex $\vec{E}_{j}$ of $S$ (which is a vector of the standard orthonormal basis of $\mathbb{R}^{n}$ ) we choose the middle points $\left(\vec{E}_{j}+\vec{E}_{i}\right) / 2$ on the edges emanating from $\vec{E}_{j}$. Now, consider the hyperplane $\Pi_{j}=\left\{r_{j}=1 / 2\right\}$ in $\Pi$ spanned by these middle points. The intersection of this plane with $S$ is a simplex. 
Now, the polyhedron $D_{n}=\pi\left(\mathcal{P}_{n}\right)$ has faces of two types:

(a) "Tetrahedral" faces which appear as intersections of $\Pi_{j}$ and $S$. These faces are combinatorially equivalent to $n-2$-simplices.

(b) "Octahedral" faces $O_{j}$ which are contained in the faces $r_{j}=0$ of the simplex $S$.

Thus all octahedral faces of $D_{n}$ are congruent to the polyhedron $D_{n-1}$.

Definition 1. A polygon $P$ in $\mathbb{E}^{2}$ is said to be degenerate if it is contained in a straight line.

The set of "boundary points" $\delta\left(\mathcal{P}_{n}\right)=\pi^{-1}\left(\partial D_{n}\right) \subset \mathcal{P}_{n}$ consists of:

(a) degenerate polygons which belong to the preimage (under $\pi$ ) of the tetrahedral faces of $D_{n}$;

(b) polygons which have one edge of length zero, they belong to the preimage (under $\pi$ ) of the octahedral faces of $D_{n}$.

Remark 2. The space $\mathcal{P}_{n}$ is compact, connected and has the Fubini-Study metric. Thus, each polygon $P \in \mathcal{P}_{n}$ can be continuously deformed to any polygon $Q \in \mathcal{P}_{n}$ so that:

the curve $\gamma=P(t)$ in $\mathcal{P}_{n}$ between $P$ and $Q$ is a geodesic segment in $\mathcal{P}_{n}$ of length not greater than a certain number $w_{n} \in \mathbb{R}$, where $w_{n}$ doesn't depend on $P, Q$.

3. Now we are interested in points $r$ of $D_{n}$ such that $\pi^{-1}(r)$ contains a degenerate polygon $P$. The set $\Sigma$ of such points is called the "critical locus" of $D_{n}$. The fact that the polygon $P$ is degenerate is equivalent to:

$$
f(r)=\sum_{j}(-1)^{u_{j}} r_{j}=0
$$

where $r_{j}$ are the lengths of the sides of $P$ and $u_{j} \in\{0,1\}$. We shall always normalize the functionals $f$ so that the number of odd exponents $u_{j}$ is not less than the number of even exponents. Therefore, the "critical locus" of $D_{n}$ is the intersection of $D_{n}$ with the union of the hyperplanes (3). Intersection of any plane (3) with $\operatorname{int}\left(D_{n}\right)$ is called a wall. The "index" ind $(W)$ of the wall $W=\{f=0\}$ is the number of even exponents $u_{j}$ in the formula (3) for the functional $f$.

Lemma 2. If $[P] \in \mathcal{P}_{n}$ is nondegenerate, then the map $\pi$ is a submersion at $[P]$.

Proof. Let $P=\left[v_{1}, \ldots, v_{n}\right]$. Then the kernel of the derivative $d \pi_{[P]}$ 
is isomorphic to the space

$$
\begin{aligned}
& Z=\left\{\xi=\left(\xi_{2}, \ldots, \xi_{n}\right) \in\left(\mathbb{R}^{2}\right)^{n-1}:\right. \\
&\left.\xi_{j} \cdot \overrightarrow{e_{j}}=0(j=2, \ldots, n), \quad \sum_{j=2}^{n} \xi_{j}=0\right\}
\end{aligned}
$$

Since $P$ is nondegenerate, the vectors $\left\{\overrightarrow{e_{j}}, j=2, \ldots, n\right\}$ span $\mathbb{R}^{2}$. Direct calculation shows that the dimension of the space $Z$ is $n-3$. The real dimension of $\mathcal{P}_{n}$ near $[P]$ is $2 n-4$. However, $2 n-4=(n-3)+n-1$, i.e.,

$$
\operatorname{dim} \operatorname{Ker}\left(d \pi_{[P]}\right)+\operatorname{dim} D_{n}=\operatorname{dim} \mathcal{P}_{n}
$$

and therefore, $\pi$ is a submersion near $[P]$.

This lemma can be also proven using the results of [17] and the connection between the space $\pi^{-1}[r]$ and a representation variety of a hyperbolic reflection group (relative to its parabolic subgroups).

4. The components of the space $D_{n}-\Sigma$ are called the chambers of $D_{n}$.

Lemma 2 implies that the moduli spaces $\pi^{-1}(r)$ are diffeomorphic for all $r$ in one and the same chamber. In treating the problem of connected components of $\pi^{-1}(r)$ the essential role is played by the great walls $W_{i j}$ which are given by (3) where $u_{j}=u_{i}=0$ and all other exponents $u_{m}$ are equal to 1 . Equivalently, $r_{i}+r_{j}=1 / 2$ on such a wall. The components of the decomposition of $D_{n}$ by great walls are called great chambers. We start with the negative great chamber where all the functionals

$$
f_{i j}=r_{i}+r_{j}-1 / 2
$$

are negative. If it exists, this chamber is called the chamber of level 0 . For each great chamber we introduce the multiindex $\mu$.

Definition 2. The great chamber $C$ has multiindex $\mu=$ $\left\{i_{1}, j_{1}\right\} \& \ldots \&\left\{i_{l}, j_{l}\right\}$ if it is given by the system of inequalities:

$$
f_{i_{1}, j_{1}}>0, \ldots . ., f_{i_{l}, j_{l}}>0 \text { and }, f_{k, s}<0 \quad \text { if } \quad\{k, s\} \notin \mu
$$

The signs $\&$ in the multiindex mean that we identify multiindices which are obtained by permutation of the pairs $\left\{i_{k}, j_{k}\right\}$. The number $l=l(C)$ here is called the level of the chamber $C$. In this case the chamber $C$ will be denoted by

$$
C_{\mu}^{n}
$$


where the index $n$ means that $C$ is a chamber in $D_{n}$. Sometimes we shall omit the upper index. If $C^{\prime} \subset C$ is a subchamber of a great chamber $C$, then the level $l\left(C^{\prime}\right)$ is by definition equal to the level $l(C)$.

5. To begin with we notice that if two couples $\{i, j\}$ and $\{k, m\}$ are presented in the multiindex of the chamber $C_{\mu}$, then either $i=k$ or $i=m$ or $j=k$ or $j=m$. Otherwise we would have:

$$
r_{i}+r_{j}>r_{k}+r_{m} \text { and } r_{i}+r_{j}<r_{k}+r_{m}
$$

in this chamber, which is impossible. Therefore, all possible multiindexes are:

(1) level 1- $\{i, j\}$;

(2) level $2-\{i, j\} \&\{i, k\}$.

(3) The set of multiindexes of the level 3 consists of 2 classes:

$$
\begin{aligned}
& \{i, j\} \&\{j, k\} \&\{k, i\} \text { (class A) } \\
& \{i, j\} \&\{i, k\} \&\{i, m\} \text { (class B) }
\end{aligned}
$$

(4) All multiindexes of level $l>3$ look like:

$$
\left\{j, i_{1}\right\} \&\left\{j, i_{2}\right\} \ldots \&\left\{j, i_{l}\right\} .
$$

The multiindexes above are said to admissible .

Proposition 1. The great chambers $C$ of level 3 and class (A) are actually chambers of $D_{n}$.

Proof. Let $C=C_{\mu}, \mu=\{i, j\} \&\{j, k\} \&\{k, i\}$. Suppose that some wall $W$ (given by (3)) intersects $C$. Then $u_{i}+u_{j}, u_{j}+u_{k}, u_{k}+u_{i}$ are odd numbers, otherwise inside $C$ we would have say $r_{i}+r_{j}<1 / 2$, which is impossible. Then the sum of these 3 odd numbers is again odd. On the other hand, the sum is equal to $2\left(u_{i}+u_{j}+u_{k}\right)$ which is even. This contradiction shows that $C$ can not intersect any wall.

Lemma 3. If $n=4$, then $D_{4}$ has only chambers of level 3, and all admissible multiindexes of level 3 are realized by great chambers in $D_{4}$.

Proof. We prove the existence of chambers applying a procedure that we shall refer to as regeneration. First consider any multiindex of class (A). Take a nondegenerate triangle $\Delta$ with sides $r_{i}, r_{j}, r_{k}$, where $i, j, k$ are different, and consider $\Delta$ as a degenerate quadrilateral where the side $r_{m}$ has zero length $(m \neq i, j, k)$. Then the inequalities (A) are 
satisfied for $\Delta$, and $\pi(\Delta)$ belongs to the face of $D_{4}$ given by equation $r_{m}=0$. Now, take the chamber of $D_{4}$ adjacent to this face. We can find an element of this chamber in the following way.

Take any positive value for $r_{m}$, which is less than

$$
\min \left\{1 / 2-r_{s}, s=i, j, k\right\} .
$$

Then there exists a quadrilateral $Q$ with the sides equal to $r_{i}, r_{j}, r_{k}, r_{m}$ ordered in some way. The perimeter $p(Q)$ of $Q$ is $1+r_{m}>1$, but we can renormalize the perimeter applying the similarity $Q \mapsto Q / p(Q)$.

All inequalities (A) are satisfied for $Q / p(Q)$, and no other inequalities of the type $f>0$ can occur. Therefore, we have an element of the chamber with the multiindex $\mu=\{i, j\} \&\{j, k\} \&\{k, i\}$.

Now, consider the case of a multiindex of class (B). Let $\Delta$ be a degenerate quadrilateral with nonzero sides $r_{i}, r_{j}, r_{k}, r_{m}$ such that $r_{i}=$ $1 / 2$. Now again find the adjacent chamber of $D_{4}$ by decreasing the length of $r_{i}$ and rescaling. Thus, we have proved the "existence" part of the Lemma. Suppose that we have a quadrilateral $Q$ with the sides $r_{1}, \ldots, r_{4}$. The set of sides breaks into pairs in 3 different ways. Suppose that $\{i, j\},\{k, m\}$ is one of these decompositions. Then we have either $r_{i}+r_{j} \geq r_{m}+r_{k}$ or $r_{i}+r_{j} \leq r_{m}+r_{k}$. In the first case we have either $f_{i, j} \geq 0$ or $f_{k, m} \geq 0$. Therefore, $\pi(Q)$ is either singular or belongs to a chamber of level 3 .

Remark 3. The polyhedron $D_{4}$ has the combinatorial type of the regular 3-dimensional octahedron which is split by the walls into 8 chambers. The walls are squares whose vertices are the vertices of the octahedron. The group $\mathbb{Z}_{4}$ (cyclic permutations of vertices of a quadrilateral) acts on $D_{4}$ preserving the chamber structure, and the action is transitive on the set of chambers of a given class.

Lemma 4. Suppose that $n \geq 5$. Then all kinds (1)-(4) of admissible multiindexes are realized by great chambers in $D_{n}$. If $n \geq 6$, then all great chambers are adjacent to the boundary of $D_{n}$.

Proof. We start the induction with the case of pentagons. The negative chamber $C_{0}$ is represented by a regular pentagon $P_{0}$. Now, for any pair $(i, j)$ consider the pentagon $P_{i j}$ where $r_{i}=r_{j}=1 / 4$, and other 3 sides have the same length $1 / 6$. Then each $\pi\left(P_{i j}\right)$ belongs to the face of $C_{0}$ contained in the hyperplane $f_{i j}=0$. Therefore, the chamber which is adjacent to $C_{0}$ along this face has the multiindex $\mu=\{i, j\}$. 
Next, for any multiindex $\{i, j\} \&\{i, k\}$ we consider a pentagon $P_{\{i j\} \&\{i k\}}$ with the sides:

$$
r_{i}=r_{j}=r_{k}=1 / 4 \text { and } r_{s}=r_{q}=1 / 8
$$

It is clear that only two of the functionals (3) are nonpositive on $\pi\left(P_{\{i j\} \&\{i k\}}\right)$. Therefore, $\pi\left(P_{\{i j\} \&\{i k\}}\right)$ belongs to the boundary of a chamber with the multiindex $\mu=\{i, j\} \&\{i, k\}$. The chambers of level 3 with multiindex $\mu$ are adjacent to the chambers of octahedral faces of $D_{5}$ which have the same multiindex $\mu$. Thus, we have established the existence for all chambers of levels 3,2 and 1. Finally, let $\mu=\{1,2\} \&\{1,3\} \&\{1,4\} \&\{1,5\}$. Take the open tetrahedral face $T_{1}$ of $\partial D_{5}$ given by the equation $r_{1}=1 / 2$. Then all inequalities $r_{1}+r_{j}>1 / 2$ are satisfied near $T_{1}$. Thus the multiindex of the chamber adjacent to $T_{1}$ is equal to $\mu$. Hence we have proved the Lemma in the case $n=5$.

Suppose now that the assertion of the lemma is proven for $n-1 \geq 5$, and we want to prove it for $n$. Let $\mu$ be any admissible multiindex which does not contain one number, say $n$ (this is equivalent to the assumption that the level of $\mu$ is less than $n-1)$. Now, consider the polyhedron $D_{n-1}$ and use the induction assumption to find a chamber $C_{\mu}^{n-1}$ with the multiindex $\mu$. Then take the chamber $C=C_{\mu}^{n}$ of $D_{n}$ which is adjacent to $C_{\mu}^{n-1}$. All inequalities (8) corresponding to the multiindex $\mu$ are satisfied in $C$, and no inequality involving $n$ like $f_{i, n}<0$ can be satisfied in $C$ since otherwise we would have : $r_{i}=1 / 2$ on $C_{\mu}^{n-1}$ which is not the case. Thus, $C$ has the multiindex $\mu$ and is adjacent to the boundary of $D_{n}$.

The last case to consider is when the multiindex has level $n-1$ :

$$
\mu=\{1,2\} \&\{1,3\} \ldots \&\{1, n\} .
$$

Take the tetrahedral face $F_{1}$ of $D_{n}$ given by the equation $r_{1}=1 / 2$, and apply the same arguments as in the case of pentagons. We conclude that the chamber $C$ adjacent to $F_{1}$ has multiindex $\mu$.

Lemma 5. Suppose that we have two chambers $C_{\alpha}, C_{\beta}$ in $D_{5}$ where the multiindex $\beta$ is obtained from $\alpha$ by adding the pair $\{i, j\}$. Then $C_{\alpha}, C_{\beta}$ have a common face at the wall $f_{i j}=0$.

Proof. Consider a generic segment $I \subset D_{5}$ which connects the interiors of these 2 chambers. Suppose that $I$ intersects some wall $f_{k m}$, where $\{k, m\} \neq\{i, j\}$. Then the inequality $f_{k m}<0$ is satisfied in 
one chamber, and $f_{k m}>0$ is satisfied in another. According to the hypothesis of lemma this implies that $\{k, m\}=\{i, j\}$.

Corollary 5. Each chamber of level 2 in $D_{5}$ is adjacent to three chambers of level 3, one of which has class $(A)$.

6. Definition 3. A chamber $C$ of $D_{n}$ is said to have type I if for some (any) point $r \in C$ the moduli space $M_{r}=\pi^{-1}(r)$ is connected. The chamber $C$ is said to be of type II if the moduli space is not connected.

Lemma 6. A chamber $C$ in $D_{n}$ has type I iff for some (any) $r \in C$ a polygon $P \in M_{r}$ can be deformed to the symmetric polygon $\sigma(P)=\bar{P}$. For any $r$ the moduli space $M_{r}$ has at most two connected components. These two components are diffeomorphic via the map $\sigma_{*}$.

Proof. We will prove the statement by induction over the number of vertices in the polygon. If $n=3$ the assertion is evident. Now, suppose that we have proved the lemma for all $k<n$. Let $P, P^{\prime}, P^{\prime \prime}$ be $n$-gons such that $\pi(P)=\pi\left(P^{\prime}\right)=\pi\left(P^{\prime \prime}\right)=r \in C$. There are two numbers $r_{i}, r_{i+1}$ in $r$ (say $i=1$ ) such that $r_{i}+r_{i+1}<1 / 2$. Therefore, we can deform $P, P^{\prime}, P^{\prime \prime}$ to polygons where 1-st and 2-nd edges belong to a straight line and their intersection is one point. Thus, we have $n-1$ gons $Q, Q^{\prime}, Q^{\prime \prime}$ with the sides : $r_{1}+r_{2}, r_{3}, \ldots, r_{n}$. However, the moduli space of $n$-1-gons consists of not more than 2 components. Thus, two of the $n$-1-gons, say $Q, Q^{\prime}$ can be deformed one to another. This means that $\pi^{-1}(r)$ consists of not more than 2 connected components.

Consider the diagonal $d$ between 1-st and 3-nd vertices of $P$. This diagonal splits $P$ into the union of a triangle $\Delta$ and an $n-1$-gon $Q$ so that $1 / 2 \geq r_{1}+r_{2}$. Applying the symmetry $\sigma$ in $d$ to the polygon $P$ we obtain another polygon $\bar{P}$ so that $\operatorname{Area}(P)=-\operatorname{Area}(\bar{P})$. We can also apply the symmetry only to $\Delta$ to obtain another polygon $N$; put $\sigma(N)=\bar{N}$.

Claim. The pairs of polygons $P, N$ and $\bar{P}, \bar{N}$ belong to the same connected components of the moduli space. Each polygon in the moduli space $M_{r}$ can be deformed either to $P$ or $\bar{P}$.

Proof. First we apply the induction hypothesis to $\operatorname{deform} Q$ to a triangle with the vertices $v_{3}, v_{4}, v_{1}$ keeping the length of $d$ fixed. Thus, we obtain a quadrilateral $Q$ with the vertices $v_{1}, v_{2}, v_{3}, v_{4}$. Now, since $r_{1}+r_{2} \leq 1 / 2$, we can deform $Q$ to a triangle $\Delta^{\prime}$ where $e_{1}, e_{2}$ belong to the straight line. We can assume that for all deformations the diagonal $d$ belongs to one and the same line $\ell=\{z \in \mathbb{C}: \operatorname{Im}(z)=0\}$. Let 
$P_{t}=g_{t}\left(\Delta^{\prime}\right)$ denote the deformation of $\Delta^{\prime}$ to $P$ where $g_{t}: \Delta^{\prime} \rightarrow P$ is a continuous family of combinatorial maps.

However, we can construct in the same way a deformation $h_{t}$ of the triangle $\Delta^{\prime}$ to the polygon $N$ as follows. If $v_{j}$ is the vertex of $\delta^{\prime}$ with $1 \leq j \leq 3$ then $h_{t}\left(v_{j}\right)=\sigma g_{t}(\sigma)$; if $j>3$ then $h_{t}\left(v_{j}\right)=g_{t}\left(v_{j}\right)$. Thus we obtain a deformation of $P$ to $N$. Application of the symmetry implies that $\bar{P}, \bar{N}$ belong to the same connected component.

However, the triangle $\Delta^{\prime}$ up to symmetry is determined only by $r$. So each polygon $P \in M_{r}$ can be deformed either to $\Delta^{\prime}$ or $\overline{\Delta^{\prime}}$.

Therefore, if $M_{r}$ has 2 connected components, no polygon $P$ can be deformed to $\bar{P}$. In this case the involution $\sigma: P \mapsto \bar{P}$ permutes the two components. Hence the lemma is proved.

7. The following is the basic property of the types of chambers.

Lemma 7. (a) Suppose that the chamber $C=C^{n}$ is adjacent to a chamber $c=C^{n-1} \subset D_{n-1}$ which has the type $X$. Then $C$ has the same type $X$. (b) If a chamber $C$ is adjacent to a tetrahedral face of $D_{n}$ then $C$ has type $I$.

Proof. Consider (a). Let $\pi(Q) \in c \subset D_{n-1}$ and

$$
Q=\lim _{t \rightarrow 0} P_{t},
$$

where $P_{t}$ is a continuous family of $n$-gons. Suppose that $C$ has type I. Then for each $t$ there exists a curve $P_{t}(s)$ between $P_{t}$ and its mirror image $\bar{P}_{t}$ so that the family of maps

$$
[0,1] \ni s \mapsto P_{t}(s)
$$

is uniformly continuous (see Remark 2). Therefore, there exists a limit

$$
\lim _{k \rightarrow \infty} P_{1 / k}(s)=Q(s),
$$

so that the continuous curve $Q(s)$ connects $Q$ with its mirror image $\bar{Q}$. Hence the chamber $c$ has type I.

Now, suppose $c$ is a chamber of type I. Let $P \in \mathcal{P}_{n-1}$ be a polygon which belongs to $\pi^{-1} c$. Then we construct a sequence of $n$-gons $Q_{k} \in$ $\mathcal{P}_{n}$ approximating $P$ so that

$$
\lim _{k \rightarrow \infty} r_{n}\left(Q_{k}\right)=0,
$$


and the sides $e_{n}, e_{n-1}$ of $Q_{k}$ belong to one and the same line. Thus we can consider the union $e_{n} \cup e_{n-1}$ as a single edge of $Q_{k}$, and $Q_{k}$ become elements $P_{k}$ of $\mathcal{P}_{n-1}$. However, the chambers of $D_{n-1}$ are open, so $P_{k} \in c$ for large $k$. Therefore, each $P_{k}$ can be deformed to its mirror image $\bar{P}_{k}$, and $Q_{k}$ belongs to the chamber of type I.

Hence, we have proved the assertion (a) of the Lemma.

First we prove the assertion (b) for $n=4$. Let $P$ be a degenerate trapezoid where $r_{1}=r_{3}, r_{4}=1 / 2$. Consider a trapezoid $Q$ sufficiently close $P$ so that for $Q$ we have:

$$
r_{4}>r_{2}, r_{4}>r_{1}+r_{2}, r_{1}=r_{3} .
$$

In this case we first can deform $Q$ to a triangle $T$ where $e_{1}$ and $e_{2}$ form a single side (Figure 1). Then we deform $T$ to the "butterfly" $B$ where the point of intersection $e_{4} \cap e_{2}$ is the center of symmetry (Figure 1). Finally, we deform $B$ to the triangle $\bar{S}$ which can be deformed to the trapezoid $\bar{Q}$ (Figure 1). This proves that the chamber $C$ has type I.

Suppose now that $n \geq 5$ and $P$ is a degenerate polygon such that $r_{1}=1 / 2$; then we can organize the edges $e_{3}, \ldots, e_{n}$ to a single edge. As the result we have a degenerate quadrilateral $Q$ and for any $Q^{\prime}$ sufficiently close to $Q$, there is a deformation of $Q^{\prime}$ to $\overline{Q^{\prime}}$. But $Q^{\prime}$ can be considered also as an element $P^{\prime}$ of $\mathcal{P}_{n}$. Therefore, $\pi\left(P^{\prime}\right)$ belongs to the chamber $C$ of the type I. This proves the assertion.

Corollary 7. The chambers of type $I$ in $D_{4}$ are the chambers of level 3 and class $(B)$; the chambers of type II in $D_{4}$ are the chambers of level 3 and class $(A)$.

Proof. The chambers of class (B) are adjacent to the "tetrahedral" faces $T_{j}$ of $D_{4}$ given by the equations $r_{j}=1 / 2$. Thus, they have the type I. The chambers of class (A) are adjacent to the "octahedral" faces of $D_{4}$ which are the polyhedrons $D_{3}$. However, the moduli space for each nondegenerate triangle consists of 2 points. Therefore, chambers of class (A) have type II.

Corollary 8. Suppose that two chambers of $D_{4}$ are adjacent along a wall $W$ given by the equation $f=0$. Then the chamber contained in the subspace $f<0$ has type $I$.

Proof. This follows from the description of the types of chambers in Corollary 7.

8. Lemma 8. Let $n \geq 5$. Suppose that two chambers are adjacent along a wall $W$ given by the equation $f=0$. If $W$ is a great wall (i.e., 
$\left.f=f_{i j}\right)$, then the chamber contained in the half-space $f<0$ has type I.

Proof. We prove all assertions of the Lemma by induction. If $n=4$ then we have already proved all assertions (Corollary 8). Suppose that we have proved the assertion for $n-1$. Let $P$ be a degenerate polygon such that $\pi(P) \in W$. Then there are at least two adjacent edges $e_{s}, e_{s+1}$ of $P$ such that their indices are different from $i, j$. Define the new polygon $Q$ with $n-1$ edges obtained from $P$ by considering $e_{s}, e_{s+1}$ as a single edge. Now, the assertion follows from the induction hypothesis applied to $Q$.

Lemma 9. Suppose that two chambers are adjacent along a wall $W$ given by the equation $f=0$. If $W$ is not a great wall, then both chambers have type I.

Proof. We prove the assertion of the lemma by induction. If $n \leq 5$ then the lemma is trivially correct. Now suppose that the assertion holds for all $k<n$. If $r=\pi(P) \in W$ does not belong to any other wall, then $P \subset \mathbb{R}=\ell$, and the image of $P$ is a closed interval with the end-points $V_{1}, V_{j}$ which are vertices of $P$. Split $P$ along the diagonal $d$ between $V_{1}, V_{j}$ into $L_{1} \cup L_{2}$.

Remark 4. It can not happen that $d$ is a side of $P$ since that would mean that the wall $W$ is a boundary face of $D_{n}$.

We suppose that $V_{1}<V_{j}$ on $\mathbb{R}$, and the edge $\left[V_{1}, V_{2}\right]$ with length $r_{1}$ is directed in the positive direction on $\mathbb{R}$. We also assume that $r_{1}$ enters equation (3) of $W$ with a positive sign. We call $\beta_{1}$ the number of backtracks on $L_{1}$ if this is the number of segments of $L_{1}$ which enter the equation of $W$ with a negative sign; $\beta_{2}$, the number of backtracks on $L_{2}$, is the number of edges of $L_{2}$ which enter the equation of $W$ with a positive sign.

Now, if $\max \left(\beta_{1}, \beta_{2}\right) \geq 2$ (say $\beta_{1}>1$ ) then we can apply the induction hypothesis to $L_{1} \cup d$ and thus prove that the whole neighborhood of $\pi(P)$ has type $\mathrm{I}$.

Suppose that $\beta_{1}=\beta_{2}=1$. Then either we can apply induction or both $L_{1}$ and $L_{2}$ contain exactly 3 segments. Thus, we have:

$$
d>r-1, d>r_{3}, r_{2}<r_{3}, r_{2}<r_{1}, d+r_{2}=r_{1}+r_{3} .
$$

Therefore,

$$
d+r_{2}=r_{1}+r_{3}, d+r-1>r_{2}+r_{3}, d+r_{3}>r_{2}+r_{1} .
$$


This means that if we deform $P$ so that the length of $d$ increases and $r_{1}, r_{2}, r_{3}$ are fixed, then this deformation of the quadrilateral $L_{1} \cup d$ has type I and we are done. Now, we can increase the length of $d$ by increasing negative as well as positive segments of $L_{2}$ (there are both). Thus for both deformations of $P$ where $f<0$ as well as $f>0$ we end up at a chamber of type I.

Now, assume that $L_{2}$ has no backtracks. Then $L_{2}$ consists of 2 segments and $L_{1}$ consists of 4 segments. Consider the pentagon $P^{\prime}=$ $L_{1} \cup d$. Direct calculation shows that $\pi\left(P^{\prime}\right)$ belongs to a common face of the negative chamber and a chamber of the level 1 . Therefore, any small deformation of $P^{\prime}$ (and hence of $P$ ) leads to a polygon of the type I.

Finally, we are left with the case where $\beta_{1}=\beta_{2}=0$. Consider the chamber adjacent to $W$ where $f>0$. Then we can organize the chain $L_{2}$ into a single segment ; denote the new polygon by $P^{+}$. Our deformation of $P^{+}$leads to a chamber of the type I in $D_{4}$ (see Lemma 8). Therefore, this deformation of $P$ has the type I. In the case of the chamber where $f<0$ we repeat the argument above by organizing $L_{1}$ into a single segment.

Corollary 9. In $D_{n}$ the chambers of level 3 and class (A) have type II, all chambers of the class (B) have type I.

Proof. If $n>4$, then each chamber of level 3 with the multiindex $\mu$ is adjacent to the boundary face $D_{n-1}$ along a chamber of the same multiindex (Lemma 4). Now the assertion follows by combining Lemma 8 and Corollary 7.

Lemma 10. In $D_{n}$ a chamber has type II if and only if it has level 3 and class (A).

Proof. Suppose that $C=C_{\mu}$ is a great chamber in $D_{n}$ with multiindex $\mu$ which is not a multiindex of the maximal level $n$ and is not of class (A), level 3. Then, we can always add to this multiindex a pair $(i, j)$ such that the new multiindex $\nu$ is still admissible (see the description of the multiindexes in section 5). Therefore, according to Lemma 8 and Lemma 5 the chamber $C$ has type I. If $C$ has maximal level $n$, then it is adjacent to a tetrahedral face of $D_{n}$ and according to Lemma 7 we conclude that $C$ again has the type I.

9. So we have proved

Theorem 1. In the polyhedron $D_{n}$ the chamber $C$ has type II if and only if $C$ is a great chamber of the level 3 and class (A). All other 
chambers correspond to connected moduli spaces $M_{r}$.

This theorem has the following geometric interpretation. We know that for each nondegenerate triangle $T$ with the sides $r=\left(r_{1}, r_{2}, r_{3}\right)$ the moduli space $M_{r}$ has exactly two components. If we "regenerate" this triangle to a $n$-gon $P$ which is sufficiently close to $T$ in $\mathcal{P}_{n}$, then the moduli space $M_{\rho}$ of $P$ again has exactly two connected components (here $\rho=\pi(P)$ ). According to Theorem 1 this is essentially the only way that any $n$-gon $Q$ can have nonconnected moduli space. Namely, if $Q$ has a nonconnected moduli space and $\pi(Q)$ belongs to a chamber $C$, then $Q$ can be deformed inside $\pi^{-1}(C)$ to a polygon $P$ as above.

10. Example. There is a point $r \in D_{5}$ which belongs to a chamber of type II such that:

on each component of $M_{r}$ the function Area has negative as well as positive values.

This means (contrary to our original expectations) that for all $n>4$ the function Sign $\circ$ Area fails to distinguish connected components of $M_{r}$ for some values of $r$. Nevertheless, one can prove that for $n=4$ the Area does distinguish connected components of $M_{r}$ for any $r$. We do not know any numerical invariant of polygons that can distinguish components for $n>4$.

Construction. We start with a convex polygon $P^{\prime}=A B C D$ such that:

(a) the angle at the vertex $C$ is $\pi / 2$;

(b) the side $B C$ has length $|B C|=1$ and $|A B|=1-\epsilon$;

(c) the angle between the diagonal $B D$ and the side $A B$ is $\pi / 2$, and the length of $B D$ is equal to $d$.

The numbers $d, 1>\epsilon>0$ will be specified below. See Figure 2 .

Then, fold the triangle $A B D$ along the diagonal $B D$. As the result we have the 4-gon $Q^{\prime}$. Let $Q^{\prime \prime}$ be the mirror image of $Q^{\prime}$ with respect to $C D$. Then the union of two 4-gons $Q^{\prime}, Q^{\prime \prime}$ is a pentagon $P$ (we do not consider $C$ as a vertex of $P$ ). We denote the vertices of $P$ as: $A, B, B^{\prime}, A^{\prime}, D$ (see Figure 2). We assume that the area of the triangle $B B^{\prime} D$ is negative, then the triangles $A B D, B^{\prime} A^{\prime} D$ have equal positive area.

The conditions on $d, \epsilon$ are:

$$
d^{2}(1-\epsilon)^{2}>d^{2}-1
$$




$$
(1-\epsilon)+1<\sqrt{d^{2}+(1-\epsilon)^{2}} .
$$

The first condition (24) means that the area of $B D B^{\prime}$ is less than $2 \cdot \operatorname{Area}(A B D)$, so $\operatorname{Area}(P)>0$. The second condition (25) means that $|A D|+\left|D A^{\prime}\right|>|A B|+\left|B B^{\prime}\right|+\left|B^{\prime} A^{\prime}\right|$. The inequalities (25), (24) also imply that

$$
d+\left|B B^{\prime}\right|>|A D|+|A B| .
$$

The inequalities $(25,26)$ are equivalent to:

$$
\frac{3-d^{2}}{2}<\epsilon<\frac{d-\sqrt{d^{2}-1}}{d} .
$$

However, the left side is negative if $d>2$. The right side of (27) is always positive and less than 1 . Therefore, we can find $\epsilon$ and $d$ which satisfy both conditions $(25,24)$.

The condition (26) implies that we can deform $P$ keeping the length of $B^{\prime} D$ fixed so that the quadrilateral $A D B^{\prime} B$ becomes an embedded triangle with negative area (the triangle $B D B^{\prime}$ does not degenerate under this deformation, but the triangle $A B D$ degenerates and changes the sign of area). We continue this deformation so that the triangle $D A B B^{\prime}$ doesn't change the area, but the triangle $D A^{\prime} B^{\prime}$ degenerates and changes the sign of area). As the result we have an embedded pentagon $Q$ where all vertices except $D$ belong to a straight line. The area of this pentagon is negative.

Finally, we can normalize the perimeter of the polygon $P$ by applying a similarity. If the image $\pi(P)$ is the vector $r$, then direct calculation shows that the point $r$ belongs to the chamber of class (A), level 3. Therefore, the moduli space $M_{r}$ is not connected, but the function Area has both negative and positive values in one component of $M_{r}$.

11. Now, we consider the global topology of the moduli spaces $M_{r}$ for nonsingular $r$. Since the projection $\pi$ is a trivial fibration near $M_{r}$, if the fiber $M_{r}$ is not orientable, then for some small neighborhood $U$ of $r$ the priemage $\pi^{-1}(U)=\mathbb{R}^{n-1} \times M_{r}$ is nonorientable as well. Therefore, the space $\mathcal{P}_{n}$ is not orientable which contradicts the fact that $\mathcal{P}_{n}=\mathbb{C} P^{n-2}$. This contradiction shows that all regular fibers $M_{r}$ are orientable. For $r \in \Sigma$ the moduli space $M_{r}$ is always singular (the singularities are isolated and correspond to degenerate polygons). These singularities 
are always quadratic. Each singular point $P$ is an isolated fixed point of the involution $\sigma_{*}: M_{r} \rightarrow M_{r}$. If a point $P$ separates its neighborhood $U$ in $M_{r}$, then $\sigma_{*}$ interchanges the two connected components of $U-P$, and $\pi(P)$ belongs to a great wall. We shall discuss the singularities in detail in our forthcoming paper.

12. Our next problem is to understand how the topology of the moduli space $M_{r}$ changes when the parameter $r$ "crosses a wall" in $D_{n}$. We recall that the space $\mathcal{P}_{n}$ is a hyperplane section in the space of "free linkages" $\tilde{\mathcal{P}}_{n}=\mathbb{C} P^{n-1}$. We will think about the space $\tilde{\mathcal{P}}_{n}$ as the configuration space of ordered $n+1$-tuples $\left(v_{0}, \ldots, v_{n}\right)$ in $\mathbb{C}^{n+1}$ modulo the diagonal action of the group of similarities $\operatorname{Aff}(\mathbb{C})$. The edges of the free linkage $z=\left(v_{0}, \ldots, v_{n}\right)$ are the vectors $e_{j}=v_{j}-v_{j-1}$. We denote by $\tilde{\pi}$ the projection

$$
\tilde{\pi}: z=\left(e_{1}, \ldots, e_{n}\right) \mapsto\left(r_{1}=\left|e_{1}\right|, \ldots, r_{n}=\left|e_{n}\right|\right)
$$

for any point $z \in \tilde{\mathcal{P}}_{n}$ with the normalized perimeter:

$$
\left|e_{1}\right|+\ldots+\left|e_{n}\right|=1 .
$$

Then $F_{r}=\tilde{\pi}^{-1}(r)$ is the space of free linkages with fixed side lengths modulo the action of the group of similarities $\operatorname{Aff}(\mathbb{C})$. We consider the smooth function $h: F_{r} \rightarrow \mathbb{R}$ given by

$$
h\left(e_{1}, \ldots, e_{n}\right)=\left|e_{1}+\ldots+e_{n}\right|^{2}=d^{2}\left(v_{0}, v_{n}\right) .
$$

We let $E_{r}$ be the complement $F_{r} \backslash h^{-1}(0)$. In what follows we will use $\mathbb{S}^{1}\left(r_{i}\right)$ to denote the circle in $\mathbb{C}$ with center at 0 and radius $r_{i}$. Let $N_{r}=\mathbb{S}^{1}\left(r_{1}\right) \times \ldots \times \mathbb{S}^{1}\left(r_{n}\right)$. Then $F_{r}$ is the quotient of $N_{r}$ by $S O(2)$ acting diagonally. We next let $X_{r} \subset N_{r}$ be the smooth submanifold defined by

$$
\begin{aligned}
X_{r}=\left\{\left(e_{1}, \ldots, e_{n}\right) \in N_{r}: v_{n}\right. & \in \mathbb{R}_{+}, \\
\text {i.e., } \operatorname{Im}\left(v_{n}\right) & \left.=0, \operatorname{Re}\left(v_{n}\right)>0\right\}
\end{aligned}
$$

Then $X_{r}$ is a slice for the projection $N_{r} \rightarrow F_{r}$ over $E_{r}$. Suppose that $\Lambda \in E_{r}$ is a degenerate configuration, i.e., all points of $\Lambda$ belong to a straight line $L$.

Define $f=f(\Lambda)$ to be the number of edges $e_{i}$ that point in the direction $\overrightarrow{v_{0} v_{n}}=\vec{d}(f$ is the number of "forwardtracks"), and $b=b(\Lambda)$ 
to be the number of edges $e_{j}$ that point toward the direction $-\vec{d}$ (the number of "backtracks"). We shall assume that the line $L$ which contains $\Lambda$ is $\mathbb{R}=\{z: \operatorname{Im}(z)=0\} \subset \mathbb{C}, v_{0}=0$ and $v_{n} \in \mathbb{R}_{4}$.

Lemma 11. (i) The function $h$ is a Morse function on $E_{r}$. (ii) The Hessian of $h$ at any degenerate configuration $\Lambda$ has $b(\Lambda)$ positive eigenvalues and $f(\Lambda)-1$ negative eigenvalues.

Proof. We shall identify the slice $X=X_{r}$ with $E_{r}$. We define two functions $g, k$ on $N_{r}$ by

$$
\begin{aligned}
& k\left(e_{1}, \ldots, e_{n}\right)=\sum_{j=1}^{n} \operatorname{Re}\left(e_{j}\right), \\
& g\left(e_{1}, \ldots, e_{n}\right)=\sum_{j=1}^{n} \operatorname{Im}\left(e_{j}\right) .
\end{aligned}
$$

We observe that $X_{r}=g^{-1}(0) \cap k^{-1}\left(R_{+}\right)$. If $\Lambda \in X_{r}$ then $g$ is a smooth function near $\Lambda$. We note that $\left.h\right|_{X}=k^{2}$, and since $k>0$ on $X$ (by definition) we may replace $h$ by $k$. We first study the critical point behavior of $k$ on $N_{r}$. Let $(r, \theta)$ be the polar coordinates in $\mathbb{C}$. Hence

$$
k\left(\left(r_{1}, \theta_{1}\right), \ldots,\left(r_{n}, \theta_{n}\right)\right)=\sum_{j=1}^{n} r_{j} \cos \theta_{j} .
$$

It is immediate that $\Lambda^{*} \in N_{r}$ is a critical point of $k$ if and only if $\Lambda^{*}$ is degenerate (i.e., contained in the real line $\mathbb{R}$ ). We shall use the vectorfields $\frac{\partial}{\partial \theta_{1}}, \ldots, \frac{\partial}{\partial \theta_{n}}$ to define coordinates on the tangent bundle of $X$. Then the matrix representation of the Hessian $\left.D^{2} k\right|_{\Lambda^{*}}$ of the function $k$ is

$$
\left[\begin{array}{cccc}
-\epsilon_{1} r_{1} & 0 & \ldots & 0 \\
0 & -\epsilon_{2} r_{2} & \ldots & 0 \\
\vdots & \vdots & \ddots & \vdots \\
0 & 0 & \ldots & -\epsilon_{n} r_{n}
\end{array}\right]
$$

where $\epsilon_{j}=1$ if $e_{j}$ is a forward track and $\epsilon_{j}=-1$ otherwise. Hence the Hessian $\left.D^{2} k\right|_{\Lambda^{*}}$ has signature $(b, f)$.

We claim that critical points of $\left.k\right|_{X}$ coincide with the critical points of $k$ which lie on $X$. Indeed, $\left.k\right|_{X}$ has a critical point at $\Lambda$ if and only 
if there exists $\lambda \in \mathbb{R}$ such that $\left.d k\right|_{\Lambda}=\left.\lambda d g\right|_{\Lambda}$. Hence if $\left(\theta_{1}, \ldots, \theta_{n}\right)$ are coordinates of $\Lambda$, then we have $\lambda=\tan \theta_{j}$ for all $j=1, \ldots, n$. Therefore either $\theta_{j}=\theta_{i}$ or $\theta_{j}= \pm \pi+\theta_{i}$. This means that $\Lambda$ is contained in $\mathbb{R}$ and $\lambda=0$. Thus the claim follows.

We now investigate the Hessian of $\left.k\right|_{X}$ at a critical point $\Lambda^{*}$. We put $B=\left.\left(D^{2} k\right)\right|_{\Lambda^{*}}$. Since the submanifold $X \subset N_{r}$ is defined by the equation

$$
\sum_{j=1}^{n} \operatorname{Im}\left(e_{j}\right)=\sum_{j=1}^{n} r_{j} \sin \theta_{j}=0
$$

we conclude that $T_{\Lambda^{*}}(X) \subset T_{\Lambda^{*}}\left(N_{r}\right)$ is defined by the equation

$$
\sum_{j=1}^{n} \epsilon_{j} r_{j} d \theta_{j}=0
$$

Hence using the basis $\frac{\partial}{\partial \theta_{1}}, \ldots, \frac{\partial}{\partial \theta_{n}}$ we find that

$$
T_{\Lambda^{*}}(X)=\left\{\left(c_{1}, \ldots, c_{n}\right) \in \mathbb{R}^{n}: \sum_{j=1}^{n} \epsilon_{j} r_{j} c_{j}=0\right\} .
$$

Therefore, $T_{\Lambda^{*}}(X)$ is the orthogonal complement of the vector $\Delta=$ $(1, \ldots, 1)$ for the quadratic form $B$. However,

$$
B(\Delta, \Delta)=-\sum_{j=1}^{n} \epsilon_{j} r_{j}=-d\left(v_{0}, v_{n}\right)
$$

Since $B(\Delta, \Delta)<0$ we find that the signature of the restriction of $B$ on $T_{\Lambda^{*}}(X)$ is $(b, f-1)$.

12. We restrict ourselves now to the cases $n=4, n=5, n=6$.

Suppose that $n=4$ and $r \in C_{\mu}$ which is a chamber of class (A). Then the moduli space $M_{r}$ is a union of two disjoint smooth circles. If $C_{\mu}$ has class (B), then $M_{r}$ is a smooth circle. For each $r \in \Sigma\left(D_{n}\right)$ the configuration space $M_{r}$ is connected and has singularities. If $r$ is a point of a wall $W$ in int $\left(D_{4}\right)$ which does not belong to any other wall, then $M_{r}$ contains a unique degenerate polygon. Therefore $M_{r}$ is a bouquet of two circles, and the only singular point is the point of intersection of two circles. Suppose now that $r$ belongs to the intersection of exactly 2 walls. Then $M_{r}$ has two singular points. In this case (up to renumeration) we have either $r_{1}=r_{3}, r_{2}=r_{4}$ or $r_{1}=r_{2}, r_{3}=r_{4}$. 
Neither of these two points can separate $M_{r}$. Therefore, the moduli space is the union of 2 circles identified at two different points. Finally, we have the case of the triple intersection point (rhombus). In this case we have 3 singular points on $M_{r}$; neither of them separates $M_{r}$, and thus $M_{r}$ is a cycle of 3 circles, any pair of which has a common point. See Figure 3 below.

13. Now, assume that $n=5$.

Suppose that we have either (a) two adjacent chambers $C=C_{\mu}, C^{\prime}=$ $C_{\nu}$ so that the level of $C_{\mu}$ is $l$, the level of $C_{\nu}$ is $l+1$, or (b) a chamber $C$ adjacent to a tetrahedral boundary face of $D_{5}$. Without loss of generality we can assume that in the case (a) $\{i, 5\}$ is in $\nu$ but not in $\mu$, in the case (b) the chamber $C$ is adjacent to the face $W$ given by the equation $r_{5}=1 / 2$ and we put $C^{\prime}=W$ in this case. Denote by $W=\left\{f_{i, 5}=0\right\}$ the common wall of $C, C^{\prime}$. Consider a smooth path $\gamma(t)$ so that $\gamma(a) \in C, \gamma(b) \in C^{\prime}$ and along this path the lengths $r_{j}$ $(j=1, \ldots, 4)$ are constant and for $r=\gamma(t)$ we have: $r_{5}=t$. We can assume that $0<a<b<\infty$. Let $s=\gamma^{-1}(W)$. Then $q=\gamma(s)$. We consider $q$ to be generic if it does not belong to any wall different from $W$.

Remark 5. In case (a) we may choose a degenerate polygon $P=\left[v_{1}, \ldots, v_{5}\right] \in \pi^{-1}(q)$ such that $v_{0}=0, v_{5} \in \mathbb{R}_{+}$. Consider the free degenerate linkage $\Lambda=\left(v_{1}, \ldots, v_{5}\right)$. Then for every generic $q$ the linkage $\Lambda$ has 1 backtrack. (Otherwise either we have case (b) and $b(\Lambda)=0$, or $b(\Lambda)=2$ and $r_{l}+r_{k}+r_{5}=1 / 2$, for some $l, k$ such that $1 \leq l<k<5$.)

Lemma 12. For a generic choice of $q=\gamma(s)$ there exists an open neighborhood $U$ of $q$ on $\gamma$ so that:

(1) $M_{U}=\pi^{-1}(U)$ is a smooth 3-manifold and

(2) the function $r_{5}$ is a Morse function on $M_{U}$ with a nondegenerate critical value $q$ of signature $(1,2)$.

Proof. Put $\rho=\left(r_{1}, \ldots, r_{4}\right)$. Then $M_{U}$ is an open subset of the moduli space of free linkages $F_{\rho}$. Thus, by Lemma 11 , the manifold $M_{U}$ is smooth and $r_{5}$ is a Morse function. If $\Lambda$ is a degenerate linkage in $F_{\rho}$ then, by Remark $5, b(\Lambda)=1, f(\Lambda)=3$. The lemma hence follows from Lemma 11.

Now, we fix a path $\gamma$ with a generic choice of $q$.

The space $M_{q}=M_{\gamma(s)}$ is a singular surface with unique singular point $[P]$ corresponding to a degenerate pentagon $P$. Then the fact that $r_{5}$ is a Morse function near $[P]$ means that there is a small neighborhood 
$V$ of $[P]$ in $M_{\gamma}$ so that in the case (a) after some change of coordinates in $V$ the function $h=r_{5}-s$ can be written as:

$$
h(x, y, z)=-x^{2}-y^{2}+z^{2} .
$$

In the coordinates (40) we have:

$$
M_{r} \subset\{h<0\}, M_{r^{\prime}} \subset\{h>0\},
$$

since $r_{5}$ increases as we go from $C$ to $C^{\prime}$.

In the case (b) the function $h=r_{5}-s$ can be written as:

$$
h(x, y, z)=-x^{2}-y^{2}-z^{2},
$$

where $h>0$ on $M_{r}$ for each $r \notin W$. Therefore, in the case (a) the Morse surgery from $M_{r}$ to $M_{r^{\prime}}$ is equivalent to removing a handle : we first pinch a simple loop $\ell$ on $M_{r}$ to obtain $M_{q}$, and then remove the point of intersection to obtain $M_{r^{\prime}}$. If $\ell$ is homologically trivial, then $M_{r^{\prime}}$ is not connected (analogously to Lemma 8). This can happen only if $C^{\prime}$ is the chamber of the level 3, Class (A). In such a case the two components of $M_{r^{\prime}}$ are diffeomorphic; the diffeomorphism is given by the mirror reflection map $P \mapsto \bar{P}$. In other cases the loop $\ell$ is homotopically nontrivial. In the case (b) the Morse surgery is attaching a 0-handle to the connected surface $M_{r}$, and therefore, in such a case $M_{r}$ is a sphere for all $r \in C$.

Essentially the same is true for any $n$-gon. Suppose that $W$ is a wall of index $\operatorname{ind}(W)$ in $D_{n}$ given by the equation $f_{i j}=0$ where $f_{i j}=$ $r_{i}+r+j-1 / 2$ (as in formula (7)). Let $C, C^{\prime}$ be chambers adjacent to $W$ along an open subset $V$ which does not intersect any other walls, so that $f_{i j}$ is negative on $C$. Let $r \in C, p \in C^{\prime}$. Then $M_{r}$ is obtained from $M_{p}$ by a Morse surgery of the index $i n d(W)-2$. In particular, if $W$ is a great wall and $i n d(W)=2$, then $M_{r}$ is obtained from $M_{p}$ either by connected sum of two connected components of $M_{p}$ or "self-connected sum" of a single component of $M_{p}$ (attaching of a zero handle).

14. Now we can prove a theorem about the topology of $M_{r}$ for all chambers in $D_{5}$.

Theorem 2. If $r \in C$ where the chamber $C$ has the level $l$, then the Euler characteristic $\chi\left(M_{r}\right)$ is given by

$$
\chi\left(M_{r}\right)=2(l-3) .
$$


Proof. We recall that all configuration spaces $M_{r}$ are orientable and begin a level by level consideration.

Level 4. Suppose that $C$ has level 4. Apply Lemma 12 to a generic path $\gamma$ so that $\gamma(b) \in W=\left\{r_{5}=1 / 2\right\}$. The preimage $r_{5}^{-1}(1 / 2) \subset M_{U}$ consists of a single point which is a degenerate pentagon. Therefore, Lemma 11 implies that $M_{r}$ is a sphere for all $r \in \pi(\gamma)$. Thus, $M_{r}$ is a sphere for each $r \in C$.

Level 3, Class (B). Each chamber $C$ of level 3 class (B) is adjacent to some chamber $C^{\prime}$ of level 4 . Then for each $r \in C$ the connected surface $M_{r}$ is obtained from the sphere by attaching a handle, and thus $M_{r}$ is a torus.

Level 2. Each chamber $C$ of level two is adjacent to some chamber $C^{\prime}$ of level 3, class (B). Then, for each $r \in C$ the connected surface $M_{r}$ is obtained from the torus by attaching a handle, and thus $M_{r}$ is a surface of genus 2 .

Level 3, Class (A). On the other hand, each chamber $C^{\prime}$ of level 3 , class (A) is adjacent to some chamber $C$ of level 2. Thus, for each $r^{\prime} \in C^{\prime}$ the surface $M_{r^{\prime}}$ is obtained from the surface of genus 2 by a surgery along a homologically trivial loop $\ell$. The loop $\ell$ can not be homotopically trivial, otherwise $M_{r^{\prime}}$ would be the union of two nonhomeomorphic surfaces (a sphere and a surface of genus 2), which is impossible by Lemma 6 . The only possible case is that $M_{r^{\prime}}$ is the union of two tori.

We repeat the same arguments as above to prove that if the level of $C$ is 1 , then $M_{r}$ is a surface of genus 3; if $C$ has level 0 , the $M_{r}$ is a surface of genus 4 . This concludes the proof of Theorem 2 .

15. Our next problem is to describe the topology of the moduli spaces of hexagons. Let $r \in D_{n}$; denote by $M_{r}^{\prime}$ the space of $n$-gons in $\mathbb{C}$ with the side lengths $r=\left(r_{1}, \ldots, r_{n}\right)$ modulo translations. We recall that $N_{r}$ is the torus

$$
\left\{e=\left(e_{1}, \ldots, e_{n}\right) \in \mathbb{C}^{n}:\left|e_{j}\right|=r_{j}, j=1, \ldots, n\right\} .
$$

We define the momentum map $\sigma: N_{r} \rightarrow \mathbb{C}$ by

$$
\sigma\left(e_{1}, \ldots, e_{n}\right)=e_{1}+\ldots+e_{n} .
$$

Then $M_{r}^{\prime}$ is canonically isomorphic to $\sigma^{-1}(0)$ by sending $\left(v_{1}, \ldots, v_{n}\right)$ to $\left(v_{2}-v_{1}, \ldots, v_{1}-v_{n}\right)$. It is easily seen that 0 is a regular value of $\sigma$ 
provided $r$ is not on a wall of $D_{n}$. As in Lemma 11 we define two functions $k, g$ on $N_{r}$ by

$$
\begin{aligned}
& k\left(e_{1}, \ldots, e_{n}\right)=\sum_{j=1}^{n} \operatorname{Re}\left(e_{j}\right), \\
& g\left(e_{1}, \ldots, e_{n}\right)=\sum_{j=1}^{n} \operatorname{Im}\left(e_{j}\right) .
\end{aligned}
$$

We let $Y \subset N_{r}$ be the zero level set of $g$. Then $Y$ is smooth. We wish to describe the level sets of $h=\left.k\right|_{Y}$ near zero. Let $\hat{r}=\left(r_{1}, \ldots, r_{n}, \epsilon\right) \in$ $D_{n+1}$.

Lemma 13. (i) $h^{-1}(0)=M_{r}^{\prime}$;

(ii) for $\epsilon>0, h^{-1}(\epsilon)=M_{\hat{r}}$ is the space of $n+1$-gons with side lengths $\left(r_{1}, \ldots, r_{n}, \epsilon\right)$ modulo the action of $\operatorname{Aff}(\mathbb{C})$.

Proof. We observe that $|h|=\left.r_{n+1}\right|_{Y}$ where $r_{n+1}=\sqrt{k^{2}+g^{2}}$, since $g \equiv 0$ on $Y$. If $\left(e_{1}, \ldots, e_{n}\right) \in h^{-1}(\epsilon)$, then we put $e_{n+1}=-\left(e_{1}+\ldots+e_{n}\right)$ so that $\left|e_{n+1}\right|=\epsilon$. Thus, we obtain an $n+1$-gon with side lengths $\left(r_{1}, \ldots, r_{n}, \epsilon\right)$ so that $v_{n+1}=\epsilon \in \mathbb{C}$. The set of these $n+1$-gons is a cross-section to the action of $\operatorname{Isom}_{+}(\mathbb{C})$ on $M_{\hat{r}}^{\prime}$ and the lemma follows.

Lemma 14. Zero is not a critical value of the function $h$.

Proof. It suffices to show that $\left.d k\right|_{Q}$ and $\left.d g\right|_{Q}$ are linearly independent (over $\mathbb{R}$ ) functionals on $T_{Q}\left(N_{r}\right)$ for $Q \in k^{-1}(0)$. Since $\sigma=k+\sqrt{-1} g$ the lemma follows from the preceding observation that 0 is a regular value for $q$.

As a consequence of the previous two lemmas we obtain the following.

Corollary 15. Suppose that $r=\left(r_{1}, \ldots, r_{n}\right) \in \operatorname{int}\left(D_{n}\right)$ does not lie on any wall, and let $\hat{r}=\left(r_{1}, \ldots, r_{n}, \epsilon\right)$. Then for sufficiently small positive $\epsilon$ the space $M_{\hat{r}}$ is diffeomorphic to $M_{r} \times \mathbb{S}^{1}$.

Proof. Clearly we have $M_{r}^{\prime} \cong M_{r} \times \mathbb{S}^{1}$. On the other hand, Lemma 14 implies that the fibers $h^{-1}(\epsilon)$ are diffeomorphic to $h^{-1}(0)$ for small positive $\epsilon$. Therefore, by Lemma 13 we conclude that $M_{\hat{r}}$ is diffeomorphic to $M_{r} \times \mathbb{S}^{1}$.

Consider now the cell structure of $D_{6}$. The walls in $D_{6}$ are either great walls or walls of index 3 . All hyperplanes defining the walls of index 3 contain the center of $D_{6}$ which is the point $\mathcal{O}=$ $(1 / 6,1 / 6,1 / 6,1 / 6,1 / 6,1 / 6)$ represented by the regular hexagon. Therefore, each component of the decomposition of $D_{6}$ by walls of index 3 is 
a cone with center at $\mathcal{O}$ over a tetrahedral face of $\partial D_{6}$ or over a chamber of an octahedral face. We know that crossing a great wall (which decreases the level of a great chamber) results in an index zero surgery on the moduli space $M_{r}$. So, it is enough to consider the topology of the space $M_{r}$ for $r$ sufficiently close to chambers of the octahedral faces of $D_{6}$.

Suppose that $C^{\prime}=C_{\mu}^{\prime}$ is a chamber of the octahedral face of $\partial D_{6}$ given by the equation $r_{s}=0$; let $\operatorname{Cone}_{\mathcal{O}}\left(C^{\prime}\right)$ be the adjacent component of the decomposition of $D_{6}$ by walls of index 3 . Since all functionals $f_{i j}$ (see (7)) are negative at the point $\mathcal{O}$, the wall $\left\{f_{i j}=0\right\}$ intersects Cone $_{\mathcal{O}}\left(C^{\prime}\right)$ if and only if the functional $f_{i j}$ is positive on $C^{\prime}$; in particular the index $s$ is not present in the multiindex $\mu$. This means that the number of great walls intersecting $\operatorname{Cone}_{\mathcal{O}}\left(C^{\prime}\right)$ is equal to the level $l(\mu)$.

(a) First we consider the case where $C^{\prime}$ has type I. Then for $r^{\prime} \in C^{\prime}$ the moduli space of pentagons $M_{r^{\prime}}$ is an oriented compact surface $\Sigma_{g}$ of genus $g \leq 4$. If $C$ is a chamber in $C o n e_{\mathcal{O}}\left(C^{\prime}\right)$ adjacent to $C^{\prime}$, then for each $r \in C$ the moduli space of hexagons $M_{r}$ is diffeomorphic to $\mathbb{S}^{1} \times \Sigma_{g}$ (according to Corollary 15). Therefore, for $r \in \hat{C} \subset$ Cone $_{\mathcal{O}}\left(C^{\prime}\right)$ the moduli space $M_{r}$ is a connected sum of of $\mathbb{S}^{1} \times \Sigma_{g}$ and $k=l(\mu)-l(\hat{C}) \leq$ $l\left(C^{\prime}\right) \leq 4$ copies of $\mathbb{S}^{2} \times \mathbb{S}^{1}$, where $l(\hat{C})$ is the level of $\hat{C}$.

(b) Now we consider the case where $C^{\prime}$ has type II. If $C$ is a chamber in $C$ nene $_{\mathcal{O}}\left(C^{\prime}\right)$ adjacent to $C^{\prime}$, then for each $r \in C$ the moduli space of hexagons $M_{r}$ is diffeomorphic to the disjoint union of two copies of $T^{3}=\mathbb{S}^{1} \times \mathbb{S}^{1} \times \mathbb{S}^{1}$. Suppose that $\hat{C}$ is a chamber in $C o n e_{\mathcal{O}}\left(C^{\prime}\right)$ which is adjacent to $C$. Then, according to Lemma 8 , the chamber $\hat{C}$ must have type I; therefore, for each $r \in \hat{C}$ the moduli space $M_{r}$ is diffeomorphic to the connected sum $T^{3} \# T^{3}$. The chamber $C^{\prime}$ has level 3. Hence, for all other chambers $\tilde{C}$ in $C_{\text {one }}\left(C^{\prime}\right)$ the moduli spaces $M_{r}$ are diffeomorphic to connected sums of $T^{3} \# T^{3}$ and $t$ copies of $\mathbb{S}^{2} \times \mathbb{S}^{1}$, where $t$ is equal to $3-l(\tilde{C}) \leq 2$.

(c) The last case is when $C^{\prime}$ is a tetrahedral face of $\partial D_{6}$. Then 5 great walls intersect $C_{0 n e_{\mathcal{O}}}\left(C^{\prime}\right)$. If $C$ is a chamber of $D_{6}$ adjacent to $C^{\prime}$, then the moduli space $M_{r}$ is diffeomorphic to $\mathbb{S}^{3}$ for all $r \in C$. If $\hat{C}$ is any chamber in $\operatorname{Cone}_{\mathcal{O}}\left(C^{\prime}\right)$ and $r \in \hat{C}$, then $M_{r}$ is diffeomorphic to the connected sum of $5-l(\hat{C})$ copies of $\mathbb{S}^{2} \times \mathbb{S}^{1}$.

Thus we have proved

Theorem 3. If the moduli space of hexagons $M_{r}$ is nonsingular and 
connected, then it is either diffeomorphic to a connected sum of $k$ copies of $\mathbb{S}^{2} \times \mathbb{S}^{1}$ and of the product $\Sigma_{g} \times \mathbb{S}^{1}$, or it is diffeomorphic to connected sum of $T^{3} \# T^{3}$ and $t \leq 2$ copies of $\mathbb{S}^{2} \times \mathbb{S}^{1}$. Here $r$ belongs to a chamber $C$, the genus $g$ of the surface $\Sigma_{g}$ is not greater than 4 and $k \leq 4$. If $M_{r}$ is nonsingular but is not connected, then it is homeomorphic to the disjoint union of two copies of $\mathbb{S}^{1} \times \mathbb{S}^{1} \times \mathbb{S}^{1}$.

16. Stable measures on the unit circle.

Let $D$ be the unit disc in the complex plane centered at zero. Denote by $\mathbb{S}^{1}$ the boundary of $D$. We identify the group of conformal automorphisms $\operatorname{Conf}(D)$ of the disc $D$ with the connected component $S O(2,1)_{0}$ of $S O(2,1)$.

The following definition is motivated by Mumford's notion of stable points for actions of semi-simple Lie groups on algebraic varieties.

Definition 4. A probability measure $\mu$ on $\mathbb{S}^{1}$ is said to be stable if the mass of any atom of $\mu$ is less than $1 / 2$. A measure is said to be semi-stable if the mass of any atom of $\mu$ is not greater than $1 / 2$. We recall that the center of mass of a probability measure $\mu$ on $\mathbb{S}^{1}$ is equal to

$$
B(\mu)=\int_{\mathbb{S}^{1}} z d \mu(z) .
$$

Theorem 4. For each stable measure $\mu$ on $\mathbb{S}^{1}$ there exists a conformal transformation $\gamma \in \operatorname{Conf}(D)$ such that $B\left(\gamma_{*} \mu\right)=0$. The element $\gamma$ is unique up to the postcomposition $g \circ \gamma$ where $g$ belongs to the orthogonal group $S O(2)$ which is the stabilizer of 0 in Conf $(D)$.

Proof. Douady and Earle in [5] define the conformal center of mass $C(\mu) \in D$ for any stable probability measure $\mu$ on $\mathbb{S}^{1}$. The point $C(\mu)$ has the following properties:

(a) For any $\gamma \in \operatorname{Conf}(D)$

$$
C\left(\gamma_{*} \mu\right)=\gamma(C(\mu)) .
$$

(b) $B(\mu)=0$ if and only if $C(\mu)=0$.

Now Theorem 4 follows from transitivity of the action of $\operatorname{Conf}(D)$ on $D$.

17. Consider a stable atomic probability measure $\mu$ on $\mathbb{S}^{1}$ which is equal to

$$
\sum_{j=1}^{k} \mu_{j} \delta_{z_{j}}
$$


where $\delta_{z}$ is the Dirac measure concentrated at the point $z \in \mathbb{S}^{1}$. We assume that all the points $z_{j}$ are distinct. There exists a unique (up to multiplication by $\left.\zeta \in \mathbb{S}^{1}\right)$ Abelian differential $\phi_{\mu} \in \Omega^{1}\left(\overline{\mathbb{C}}-\left\{z_{1}, \ldots, z_{k}\right\}\right)$ such that:

(1) the singularity of $\phi_{\mu}$ at $z_{j}$ is

$$
\left(z-z_{j}\right)^{-2 \mu_{j}} d z
$$

$$
\iint_{D}\left|\phi_{\mu}\right|^{2}=1
$$

Explicitly the differential $\phi_{\mu}$ is given by the following formula:

$$
\begin{gathered}
\omega_{\mu}=\frac{d z}{\prod_{j=1}^{k}\left(z-z_{j}\right)^{2 \mu_{j}}}, \\
\phi_{\mu}=\omega_{\mu}\left(\iint_{D}\left|\omega_{\mu}\right|^{2}\right)^{-1 / 2} .
\end{gathered}
$$

Stability of the measure $\mu$ implies that the integral $\iint_{D}\left|\omega_{\mu}\right|$ is finite and therefore the Christoffel-Schwarz map

$$
f_{\mu}: z \in D \mapsto \int_{0}^{z} \phi_{\mu}
$$

is continuous in $D \cup \mathbb{S}^{1}$ and has bounded image. It is well known that the map $f_{\mu}$ is univalent and its image is a convex polygon $P_{\mu}=f_{\mu}(D)$ in $\mathbb{C}$ with the interior angles $\alpha_{j}=\pi\left(1-2 \mu_{j}\right)$ at the vertices $w_{j}=f_{\mu}\left(z_{j}\right)$. The differential $\phi_{\mu}$ defines a Riemannian metric

$$
d s_{\mu}=\left|\phi_{\mu}\right|
$$

which has area 1 so that the map $f_{\mu}:\left(D, d s_{\mu}\right) \rightarrow \mathbb{C}$ is an isometric embedding. Suppose that $\gamma \in \operatorname{Conf}(D), \nu=\gamma_{*}(\mu)$. Then $\phi_{\nu}$ is proportional to $\gamma_{*} \phi_{\mu}$. Therefore the metrics $d s_{\nu}$ and $\gamma_{*} d s_{\mu}$ are isometric. This implies that there exists an orientation-preserving Euclidean isometry $\rho(\gamma)$ such that

$$
\rho(\gamma) \circ f_{\mu}=f_{\nu} \circ \gamma
$$




$$
\rho(\gamma)\left(P_{\mu}\right)=P_{\nu} .
$$

18. Duality between the moduli spaces of Euclidean polygons with fixed angles and Euclidean polygons with fixed side lengths.

Fix a vector $r \in D_{n}-\Sigma$, i.e., $r$ does not belong to any wall in the polyhedron $D_{n}$. Each polygon $Z$ in the moduli space $M_{r}$ corresponds to the collection of edges $\left(e_{1}, \ldots, e_{n}\right) \in\left(\mathbb{C}^{*}\right)^{n}$. The normalized vectors $z_{j}=e_{j} / r_{j}$ belong to the unit circle $\mathbb{S}^{1}$. The polygon $Z$ is defined up to Euclidean isometry; therefore the vector $\vec{z}=\left(z_{1}, \ldots, z_{n}\right)$ is defined up to rotation around zero. Since the polygon $Z$ is closed we conclude that

$$
\sum_{j=1}^{n} r_{j} z_{j}=0 .
$$

This means that we get a diffeomorphism

$$
\epsilon: M_{r} \rightarrow \mathcal{M}_{r}=\left\{\vec{z} \in\left(\mathbb{S}^{1}\right)^{n}: \sum_{j=1}^{n} r_{j} z_{j}=0\right\} / S O(2) .
$$

The vector $\vec{z}$ and collection of numbers $r=\left(r_{1}, \ldots, r_{n}\right)$ define a measure $\mu=\mu(\vec{z}, r)$ by the formula

$$
\mu=\sum_{j=1}^{n} r_{j} \delta_{z_{j}}
$$

This measure has total mass 1 and $B(\mu)=0$. The assumption $r \in$ $D_{n}-\Sigma$ implies that $\mu$ is a stable atomic measure on $\mathbb{S}^{1}$. However the number of points in the support of $\mu$ can be less than $n$. Denote by $\left\{w_{1}, \ldots, w_{k}\right\}$ the support of $\mu$ assuming that all the points $w_{j}$ are distinct. Let $\mu_{j}$ be the mass of $\mu$ in the point $w_{j}$. Then we use the formulas (54), (55) to define the Abelian differential $\phi_{\mu}$ and univalent holomorphic map $f_{\mu}: D \rightarrow \mathbb{C}$. The vector $\vec{z}$ is defined up to the action of the group $S O(2)$; therefore the map $f_{\mu}$ and the $k$-gon $P_{\mu}=f_{\mu}(D)$ are defined up to rotation in $\mathbb{C}$. The map $Z \mapsto \vec{z} \mapsto \mu \mapsto P_{\mu}$ is not injective. To deal with this problem we define marked convex $k$-gons $(P, I, b)$.

Definition 5. A marked convex $k$-gon $(P, I, b)$ is a triple where $P$ is a convex $k$-gon of area 1 in $\mathbb{C}, I$ is a partition of the set $\{1, \ldots, n\}$ 
into $k$ nonempty disjoint subsets $\left\{I_{1}, \ldots, I_{k}\right\}$, and $b$ is a bijection from $I$ onto the set of vertices of $P$. This triple must satisfy the following condition. Let

$$
\mu_{j}=\sum_{i \in I_{j}} r_{i}
$$

Then the angle of $P$ at the vertex $b\left(I_{j}\right)$ is equal to $\pi\left(1-2 \mu_{j}\right)$.

Remark 6. Note that in general the cyclic order of vertices on the boundary of $P$ has nothing to do with the bijection $b$ even if $n=k$.

We divide the space of marked $k$-gons by the action of $I s o m_{+}\left(\mathbb{E}^{2}\right)$ and denote the quotient by $E_{k}=E_{k}(r)$. The union

$$
E=E(r)=\cup_{k=3}^{n} E_{k}(r)
$$

is the space of (congruence classes) of marked convex polygons in $\mathbb{C}$ which have prescribed angles. Thus, if $[\vec{z}] \in \mathcal{M}_{r}$ then we define the corresponding marked polygon $(P, I, b)=\psi([\vec{z}]) \in E(r)$ as follows. The (congruence class) of the polygon $P$ is given by the image of the Christoffel-Schwarz map $f_{\mu}$ as above. The map from $\left(z_{1}, \ldots, z_{n}\right)$ to the support of the measure $\mu$ defines the partition $I$ of the set $\{1, \ldots, n\}$ into preimages of atoms of $\mu$. The projection $\left(z_{1}, \ldots, z_{n}\right) \mapsto \operatorname{supp}(\mu)$ also indices a bijection $b$ from $I$ to the set of vertices of $P$. It is easy to see that the composition $\tau=\psi \circ \epsilon: M_{r} \rightarrow E(r)$ is injective.

To prove that $\tau$ is surjective take any marked $k$-gon $(P, I, h)$. All angles of $P$ are less than $\pi$. There exists a Riemann mapping $f: D \rightarrow$ $P$. This map extends to a homeomorphism $D \cup \mathbb{S}^{1} \rightarrow c l P$. To define the corresponding measure $\mu$ we take the preimage of the set of vertices of $P$ to be the support of $\mu$. If $v_{j}$ is a vertex of $P$ with the angle $\alpha_{j}$, then the mass of $\mu$ at the corresponding atom $w_{j}$ is equal to

$$
\mu_{j}=\left(\pi-\alpha_{j}\right) / 2 \text {. }
$$

It follows that $\mu$ is a stable measure. Thus, Theorem 4 implies that there exists a Moebius transformation $\gamma \in \operatorname{Conf}(D)$ such that the center of mass of $\nu=\gamma_{*}(\mu)$ is zero. The polygon $P_{\nu}$ is in the same isometry class as $P=P_{\mu}$ (see Section 17). Therefore $P_{\nu} \cong P$ belongs to the image of the map $\tau$. Hence the map

$$
\tau: M_{r} \rightarrow E(r)
$$


is a bijection. The space of marked polygons has a natural topology. A sequence $\left[P_{j}, I_{j}, b_{j}\right] \in E(r)$ is convergent to $[P, I, b]$ if the following conditions are satisfied:

(0) The partitions $I_{j}$ do not change for large $j$.

(1) For some choice of representatives $\left(P_{j}, I_{j}, b_{j}\right) \in\left[P_{j}, I_{j}, b_{j}\right]$, $(P, I, b) \in[P, I, b]$ the polygons $P_{j}$ are Hausdorff convergent to $P$.

(2) The convergence of $P_{j}$ to $P$ leads to collision of some of vertices of $P_{j}$. This defines a new partition $I_{\infty}$ of $\{1, \ldots, n\}$ and a bijection $b_{\infty}:\{1, \ldots, n\} \rightarrow I_{\infty}$. We require $I_{\infty}=I$ and $b_{\infty}=b$.

It is easy to see that $E(r)$ is compact and the map $\tau: M_{r} \rightarrow E(r)$ is continuous. Therefore $\tau$ is a homeomorphism. The space $E_{n}(r)$ is an open dense subset in $E(r)$. It is the space of (marked) convex $n$ gons with fixed angles $\alpha_{j}$. If all the numbers $r_{j}$ are different, then the marking of these polygons is given by the map $j \mapsto \alpha_{j}$.

19. The space of marked polygons with fixed angles and the moduli spaces of Deligne-Mostow, Bavard-Ghys, Kojima-Yamashita and Thurston.

Deligne-Mostow in [6] and Thurston in [15] consider the moduli space $M(\alpha)$ of flat metrics on the sphere $\mathbb{S}^{2}$ with the fixed angles $2 \alpha_{j}<$ $2 \pi$ around singular points $w_{1}, \ldots, w_{n}$ (see [15]). Equivalently $M(\alpha)$ is the moduli space of configurations of marked $n$-tuples of points on $\mathbb{C} P(1)$ (see [6]). The space $M(\alpha)$ is an incomplete complex-hyperbolic manifold of the complex dimension $n-3$. This space contains several totally-geodesic real submanifolds $H_{s}$ of real dimension $n-3$. The points in $H_{s}$ are doubles of convex polygons $P$ with the fixed angles $\alpha_{j}$ so that the cyclic ordering of vertices of $P$ is the same as the cyclic ordering $\sigma$ of $\left(\alpha_{1}, \ldots, \alpha_{n}\right)$. It was proven in [14] and [3] that $H_{s}$ are convex real hyperbolic polyhedra. These papers also describe the geometry of $H_{s}$ in terms of the collection $\left(\alpha_{1}, \ldots, \alpha_{n}\right)$. The polyhedra $H_{s}$ are compact if and only if $r \notin \Sigma$, where $\alpha_{j}=\pi\left(1-2 r_{j}\right)$. The boundary points of $H_{s}$ correspond to $k$-gons $(k<n)$ obtained by collision of some of vertices of $P \in H_{s}$. From the point of view of [6] the points of $H_{s}$ correspond to configurations of $n$ distinct points on $\mathbb{S}^{1}$ with the fixed cyclic ordering. The map from this space to $\mathbb{H}_{\mathbb{C}}^{n-3}$ can be described as follows. Let $[\vec{z}]$ be any element in $\mathcal{M}_{r}$. It corresponds to a configuration of $n$ points in $\mathbb{S}^{1}$ so that some of these points can coincide. Define 
the vector

$$
\xi=\left(\int_{0}^{z_{2}} \phi_{\mu}-\int_{0}^{z_{1}} \phi_{\mu}, \ldots, \int_{0}^{z_{n-1}} \phi_{\mu}-\int_{0}^{z_{n-2}} \phi_{\mu}\right) \in \mathbb{C}^{n-2} .
$$

The space $\mathbb{C}^{n-2}$ has a Hermitian form $h$ of signature $(1, n-3)$ whose value at $\xi$ is equal to $1=\operatorname{Area}\left(P_{\mu}\right)^{2}$. The image of $\xi$ after the projectivization $\mathbb{C}^{n-2}-0 \rightarrow \mathbb{C} P^{n-3}$ belongs to a the complex-hyperbolic space $\mathbb{H}_{\mathbb{C}}^{n-3}$. This is the Deligne-Mostow map

$$
D M: \mathcal{M}_{r}=M_{r} \rightarrow \mathbb{H}_{\mathbb{C}}^{n-3}
$$

It is easy to see that this map is continuous and projects to $\bar{M}_{r}=M_{r} / \sigma$ to an injective map $\mathcal{D} M: \bar{M}_{r} \rightarrow \mathbb{H}_{\mathbb{C}}^{n-3}$. The image of $D M$ is an $(n-3)-$ dimensional compact polyhedral submanifold $S_{r}$ without boundary. $S_{r}$ splits into the union of real hyperbolic polyhedra $H_{s}$ which are adjacent along boundary faces. For example, the component $H_{1}$ where the cyclic ordering of points $z_{1}, \ldots, z_{n}$ on $\mathbb{S}^{1}$ is the same as $(1, \ldots, n)$ corresponds to the set of convex polygons in $M_{r}$, which have a fixed orientation.

Thus we have proved the following.

Theorem 4. Let $r \in \operatorname{int}\left(D_{n}\right)-\Sigma$. Then the manifold $M_{r}$ has a natural tiling by hyperbolic polyhedra. Each tile $H_{s}$ is a moduli space of marked convex $n$-gons with fixed interior angles $\pi\left(1-2 r_{1}\right), \ldots, \pi\left(1-2 r_{n}\right)$ occuring in some fixed order. Each tile is a convex hyperbolic $(n-3)$ dimensional polyhedron which is an orthoscheme (by [3]). The quotient $\bar{M}_{r}=M_{r} / \sigma$ admits an embedding $\mathcal{D} M$ into $\mathbb{H}_{\mathbb{C}}^{n-3}$ such that each tile is isometrically embedded in a totally real totally geodesic subspace. For example the deformation space of the regular pentagon is tiled by 24 regular right angled hyperbolic pentagons inducing a hyperbolic structure on the genus 4 surface.

The duality $\tau$ can be generalized to the case of $r \in \Sigma$. In this case we have to take into account also nice semi-stable measures $\mu$ on $\mathbb{S}^{1}$ : those which have exactly two atoms. Corresponding polygons $P$ in $M_{r}$ are degenerate. If $\mu$ is a nice semi-stable measure, which is not stable, then the Abelian differential $\omega_{\mu}$ (see (53)) has infinite $L^{2}$ norm, and therefore we can not find the normalized differential $\phi_{\mu}$. Nevertheless the Deligne-Mostow map $D M$ still makes sense, and the point $D M(P)$ belongs to the ideal boundary of the complex-hyperbolic space. This is an isolated ideal boundary point of $D M\left(M_{r}\right)$ (a "cusp"). For any 
$r \in \Sigma$ the corresponding polygons in $E_{n}(r)$ have parallel sides. The number of pairs of such sides is equal to the number of walls in $D_{n}$ which contain $r$. This is the number of cusps of the space $D M\left(M_{r}\right)$.

The above construction can be generalized to cover the whole moduli space $M(\alpha)$. In this case instead of $M_{r}$ one has to consider the space of polygons in $\mathbb{E}^{3}$ with fixed side lengths.

\section{Acknowledgements.}

We are grateful to Robert Bryant, Richard Hain and David Hamilton for fruitful discussions. We would like also to thank Thomas Banchoff and Robert Connelly for providing us with many of the references.

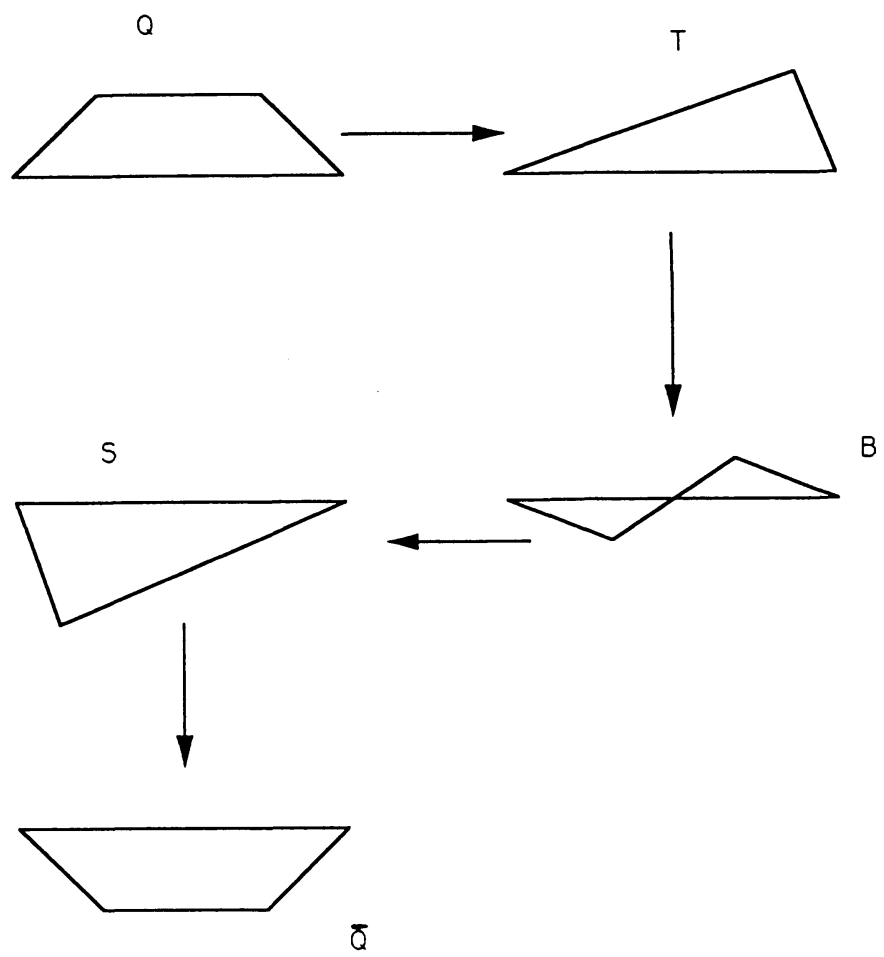

Figure 1 
MODULI SPACES OF POLYGONS

461
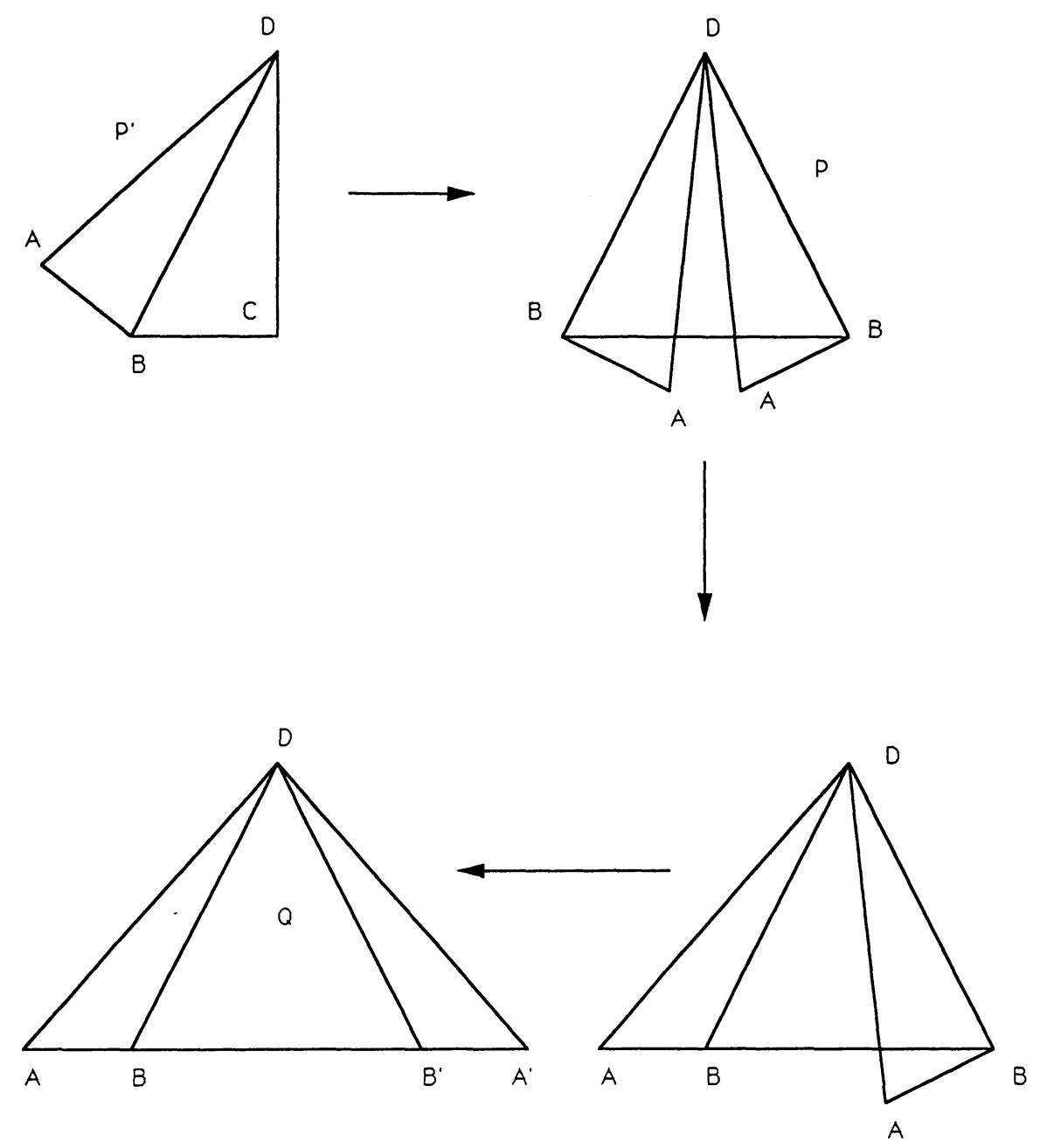

FIGURE 2 

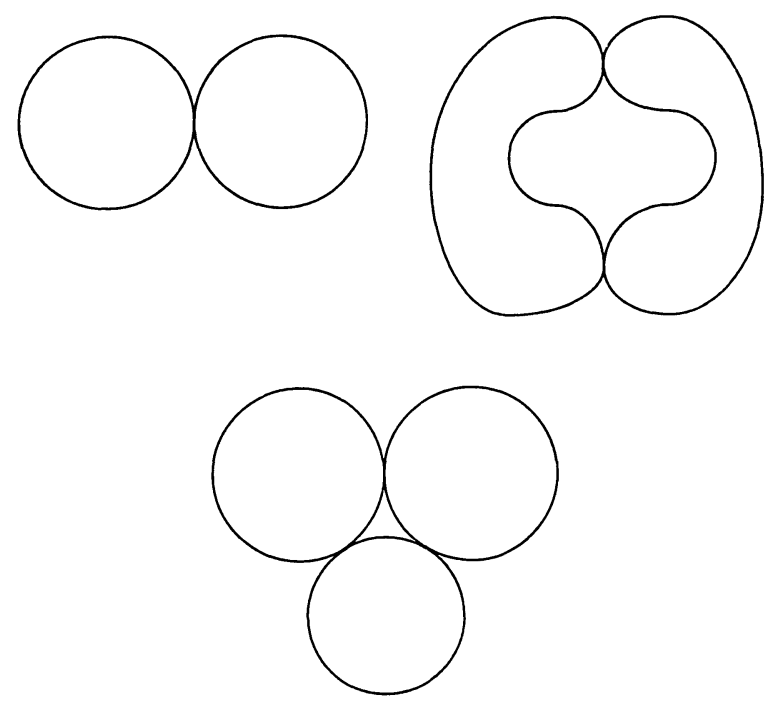

FiguRE 3
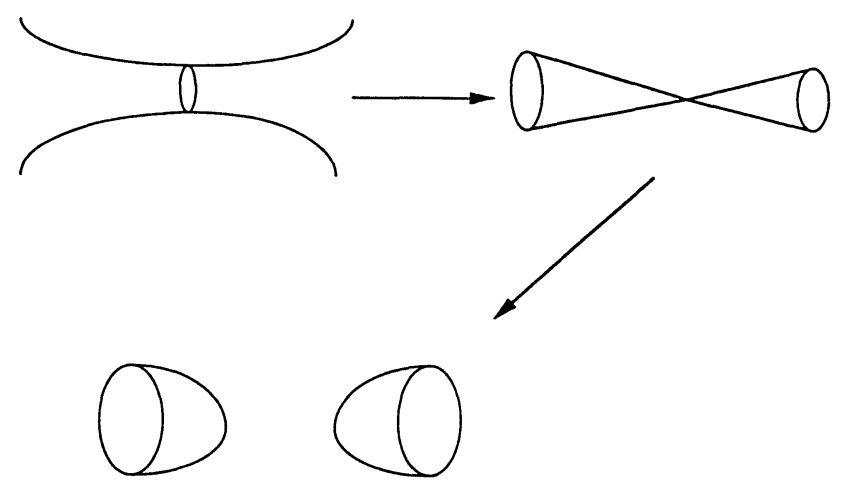

FIGURE 4 


\section{References}

[1] T.Banchoff, Relative isoperimetric problems and the topology of linkage spaces, Preprint, 1974.

[2] T.Banchoff \& P.Giblin, On the symmetry sets of piecewise circular curves, Proc. Roy. Soc. Edinburgh A 123 (1993) 1-15.

[3] C.Bavard \& E.Ghys, Polygones du plan et polyhedres hyperboliques, Geom. Dedicata 43 (1992) 207- 224.

[4] R.Connelly, personal communication, 1994.

[5] A.Douady \& C.Earle, Conformally natural extension of homeomorphisms of the circle, Acta Math. 157 (1986) 23- 48.

[6] P.Deligne \& G.Mostow, Monodromy of hypergeometric functions and non-lattice integral monodromy, Inst. Hautes Études Sci. Publ. Math. 63 (1986) 5- 90.

[7] C.G.Gibson \& D.Marsh, On the linkage varieties of watt 6-bar mechanisms, I- III, Mech. Mach. Theory 24 (1989) 106-113; 115- 121; 123- 126.

[8] _., Movable hinged spherical quadrilaterals, I, II, Mech. Mach. Theory 23 (1988) 13-18; 19-24.

[9] C.G.Gibson \& P.E.Newstead, On the geometry of the planar 4-bar mechanism, Acta Appl. Math. 7 (1986) 113- 135.

[10] J.-C.Hausmann, Sur la topologie des bras articules, (Algebraic Topology, Poznan), Lecture Notes in Math. Vol. 1474, Springer, Berlin, 1989, 146- 159.

[11] T.-F.Havel, The use of the distances as coordinates in computer-aided proofs of theorems in Euclidean geometry, J. Symbolic Comput. 11 (1991) 579-593.

[12] B.Jaggi, Configuration spaces of point sets with distance constrains, Ph. D. thesis, Univ. of Bern, 1992.

[13] Y.Kamiyama, Topology of equilateral polygon linkages, Preprint, 1992.

[14] S.Kojima \& Y.Yamashita, Shapes of stars, Proc. Amer. Math. Soc. 117 (1993) 845- 851.

[15] W.Thurston, Shapes of polyhedra, Preprint, 1987. 
[16] K.Walker, Configuration spaces of linkages, Undergraduate thesis, Princeton Univ., 1985.

[17] A.Weil, Remarks on the cohomology of groups, Ann. of Math. 80 (1964) 149 - 157.

[18] S.Whitesides, to appear in J. Discrete Comput. Geom.

\author{
UNIVERSITY OF UTAH \\ UNIVERSITY OF MARYLAND
}

